\title{
NÍVEIS DE RESÍDUO INDUSTRIAL DE FÉCULA DA MANDIOCA NA ALIMENTAÇÃO DE SUÍNOS EM CRESCIMENTO E TERMINAÇÃO'
}

\author{
TERESINHA MARISA BERTOL² e GUSTAVO JULIO MELLO MONTEIRO DE LIMA ${ }^{3}$
}

\begin{abstract}
RESUMO - Foram conduzidos dois experimentos para avaliação da inclusão do resíduo industrial de fecularia da mandioca (RIFM) em dietas para suínos em crescimento e terminação. No experimento 1 , foram avaliados quatro níveis de RIFM $(0 ; 6,67 ; 13,33$ e 20,00\%) na dieta de suínos em crescimento. A inclusão do RIFM na dieta provocou efeito cúbico sobre o ganho de peso médio diário (GPMD, $\mathrm{P}<0,01, \mathrm{R}^{2}=0,80, \mathrm{y}=804-54,06 \mathrm{x}+6,03 \mathrm{x}^{2}-0,19 \mathrm{x}^{3}$ ), peso médio ao final do crescimento (PMFC, $\mathrm{P}<0,01$, $\left.R^{2}=0,79, y=60,47-2,87 x+0,33 x^{2}-0,01 x^{3}\right)$ e efeito linear sobre o consumo diário de ração (CRD, $P<0,04$, $\left.\mathrm{R}^{2}=0,74, \mathrm{y}=2090-136 \mathrm{x}\right)$. O peso médio ao abate (PMA) foi afetado de forma quadrática $(\mathrm{P}<0,05$, $\left.\mathrm{R}^{2}=0,69, \mathrm{y}=98,88-1,28 \mathrm{x}+0,05 \mathrm{x}^{2}\right)$. No experimento 2 , o RIFM foi avaliado em quatro níveis $(0,10,20$ e 30\%) de inclusão na dieta de suínos em terminação. Nenhum desses níveis de RIFM afetou ( $\mathrm{P}>0,10)$ o desempenho dos suínos. Concluiu-se que a inclusão do RIFM na dieta de suínos em crescimento, à partir de $6,67 \%$, provoca redução do desempenho. Na fase de terminação, o desempenho não é afetado pela inclusão do RIFM até o nível de $30 \%$ da dieta.
\end{abstract}

Termos para indexação: desempenho de suínos, raspa de mandioca.

\section{LEVELS OF CASSAVA RESIDUE IN DIETS FOR GROWING AND FINISHING PIGS}

\begin{abstract}
Two experiments were conducted in order to evaluate the inclusion of cassava residue (RIFM) in diets for growing and finishing pigs. In the first experiment, four levels $(0,6.67,13.33$ and $20 \%$ ) of RIFM in growing diets were studied. The inclusion of RIFM in the diets caused a cubic effect on average daily gain (GPMD, $\mathrm{P}<0.01, \mathrm{R}^{2}=0.80, \mathrm{y}=804-54.06 \mathrm{x}+6.03 \mathrm{x}^{2}-0.19 \mathrm{x}^{3}$ ) and final body weight ( $\mathrm{PMFC}, \mathrm{P}<0.01, \mathrm{R}^{2}=0.79, \mathrm{y}=60.47-2.87 \mathrm{x}+0.33 \mathrm{x}^{2}-0.01 \mathrm{x}^{3}$ ) and a linear effect on average daily intake (CRMD, $\mathrm{P}<0.04, \mathrm{R}^{2}=0.74, \mathrm{y}=2090-136 \mathrm{x}$ ). The quadratic effect was significant for slaughter body weight (PMA, $\left.\mathrm{P}<0.05, \mathrm{R}^{2}=0.69, \mathrm{y}=98.88-1.28 \mathrm{x}+0.05 \mathrm{x}^{2}\right)$. In the second experiment, RIFM was studied in four levels $(0,10,20$ and $30 \%)$ of inclusion in diets for finishing pigs. Pig performance was not affected ( $\mathrm{P}>0.10)$ by the inclusion of any level of RIFM. It was concluded that the inclusion of RIFM in diets for growing pigs, in levels like $6.67 \%$ or higher, decrease swine performance. In the finishing phase, the performance is not affected by the inclusion of any levels of RIFM, up to $30 \%$.
\end{abstract}

Index terms: pig performance, cassava flour.

\section{INTRODUÇÃO}

A mandioca (Manihot esculenta Crantz) é uma cultura amplamente difundida no mundo, constituindose em uma das principais fontes de energia para ali-

\footnotetext{
${ }^{1}$ Aceito para publicação em 28 de julho de 1998.

Financiado pelo IAPAR - Instituto Agronômico do Paraná.

${ }^{2}$ Zoot., M.Sc., Embrapa-Centro Nacional de Pesquisa Suínos e Aves (CNPSA), Caixa Postal 21, CEP 89700-000, Concórdia, SC. E-mail: tbertol@cnpsa.embrapa.br

${ }^{3}$ Eng. Agr., Ph.D., Embrapa-CNPSA.
}

mentação humana e animal em países de clima tropical. O Brasil é um dos maiores produtores mundiais de mandioca, com o plantio dessa cultura difundido em todo o País. A maior produção está nos Estados do Pará, Paraná e Bahia, e a maior produtividade está em São Paulo, Paraná e Acre (Anuário Estatístico do Brasil, 1995).

Os principais nutrientes da mandioca são os carboidratos, altamente digestíveis, os quais estão localizados principalmente na raiz (Muller et al., 1974). O extrativo não nitrogenado representa $91,2 \%$ 
da raiz integral em base seca, e o amido representa 70 a $80 \%$ da mesma (Centro Internacional de Agricultura Tropical, 1978; Gomez, 1979). Os conteúdos de proteína, extrato etéreo, fibra bruta, minerais e vitaminas da raiz da mandioca são muito baixos. Além de o teor de proteína ser baixo, aproximadamente $50 \%$ do $\mathrm{N}$ da polpa e $70 \%$ do $\mathrm{N}$ da casca estão na forma não-protéica (Centro Internacional de Agricultura Tropical, 1970).

São muitos os subprodutos da mandioca utilizados na alimentação animal. O resíduo industrial de fecularia da mandioca (RIFM) é proveniente da extração da fécula da mandioca na indústria, apresentando $92,66 \%$ de matéria seca, $1,32 \%$ de proteína bruta (PB), 10,67\% de fibra bruta e $6,63 \%$ de cinza, de acordo com análises feitas no Laboratório de Nutrição da Embrapa-CNPSA, em Concórdia, SC. Os teores de energia digestível $(2753 \mathrm{kcal} / \mathrm{kg})$ e metabolizável $(2621 \mathrm{kcal} / \mathrm{kg})$ do RIFM para suínos são inferiores aos do milho (Embrapa, 1991).

Os resultados de desempenho obtidos em estudos anteriores nas condições brasileiras são bastante variáveis. Foi observada redução na taxa de crescimento com a utilização da raspa residual de mandioca a partir de 7,5\% e $60 \%$ (Nicolaiewsky et al., 1986a, 1986b) de inclusão na dieta de suínos em crescimento-terminação e terminação, respectivamente. Zoby et al. (1971) substituíram totalmente o milho da dieta por raspa residual de mandioca. Na fase de crescimento, esses autores observaram redução da taxa de crescimento e piora da conversão alimentar, sendo que a suplementação com metionina eliminou esses problemas, além de aumentar o consumo de alimento. Na fase de terminação, houve efeito negativo da inclusão da raspa residual de mandioca somente sobre a conversão alimentar, sendo que a suplementação com metionina não teve efeito.

O objetivo deste trabalho foi verificar a viabilidade técnica da inclusão do RIFM na dieta de suínos em crescimento e terminação.

\section{MATERIAL E MÉTODOS}

\section{Experimento 1}

Foi avaliada a inclusão de quatro níveis de RIFM na dieta de suínos em crescimento: $0 ; 6,67 ; 13,33$ e $20,00 \%$.
O experimento foi conduzido nos meses de dezembro de 1993 e janeiro de 1994, na Estação Experimental do IAPAR em Pato Branco, PR, e teve a duração de 48 dias.

Foram utilizados 32 leitões cruzados Duroc x Large White, com peso médio inicial (PMI) de 21,86 $\pm 2,22 \mathrm{~kg}$. Os animais foram distribuídos em um delineamento em quatro blocos casualizados, cada um deles com quatro tratamentos, totalizando quatro repetições por tratamento. O bloco foi caracterizado pela localização na instalação e a unidade experimental foi a baia. Cada baia continha dois animais (um macho e uma fềmea). Foram realizadas pesagens dos animais e da sobra de ração no início e no final do período experimental. Foram avaliados o ganho de peso médio diário (GPMD), consumo de ração médio diário (CRMD), conversão alimentar (CA), peso médio ao final do crescimento (PMFC) e índice nutricional bioeconômico (INBE) (Guidoni et al., 1994), sendo:

INBE $=$ GPMD - (preço da ração/preço do suíno) $\mathrm{x}$ CRMD.

Desta forma, o INBE representa o ganho de peso líquido, após serem descontados os custos com alimentação.

Após o término do experimento o peso dos animais foi acompanhado até o abate (PMA), para verificar o efeito residual dos tratamentos sobre essa variável. Durante esse período, os animais receberam uma dieta padrão de terminação, baseada em milho e farelo de soja.

Os dados foram submetidos à análise de variância pelo procedimento GLM do SAS (SAS, 1990), de acordo com o modelo matemático:

$Y_{i j}=\mu+M_{i}+B j+e_{i j}$,

$\mathrm{Y}_{\mathrm{ij}}=$ variável dependente no tratamento $\mathrm{i}$, bloco $\mathrm{j}$,

$\mu=$ média geral,

$\mathrm{M}_{\mathrm{i}}=$ efeito de tratamento ( $\left.\mathrm{i}=1,2,3,4\right)$,

$\mathrm{B}_{\mathrm{j}}=$ efeito de bloco $(\mathrm{j}=1,2,3,4)$,

$\mathrm{e}_{\mathrm{ij}}=$ erro aleatório associado a cada observação.

Os efeitos linear, quadrático e cúbico dos tratamentos (níveis de resíduo de mandioca) sobre as variáveis foram testados através de contrastes ortogonais. No tocante às variáveis em que um ou mais efeitos foram significativos, foi feita análise de regressão para obtenção das equações de regressão (SAS, 1990).

\section{Experimento 2}

Foram utilizados 48 suínos cruzados Duroc x Large White, com PMI de 54,42 $\pm 2,51 \mathrm{~kg}$, em um experimento onde foram avaliados quatro níveis de inclusão do RIFM: $0,10,20$ e $30 \%$. O experimento foi conduzido nos meses de fevereiro e março de 1994, na Estação Experimental do IAPAR em Pato Branco, PR, e teve a duração de 42 dias. 
Os animais foram distribuídos em delineamento, em seis blocos casualizados, cada um deles com quatro tratamentos, totalizando seis repetições por tratamento. O bloco foi caracterizado pela localização na instalação e a unidade experimental foi a baia. Cada baia continha dois animais (um macho e uma fêmea). Foram realizadas pesagens dos animais e da sobra de ração no início e no final do período experimental. Foram avaliados o GPMD, CRMD, CA, peso médio final (PMF) e INBE.

A análise estatística dos dados foi feita de acordo com o descrito no experimento 1 .

As dietas dos dois experimentos (Tabela 1) foram isoprotéicas e isocalóricas, variando no teor de lisina e metionina, e foram formuladas para atender ou exceder as recomendações do National Research Council (1988). A água e a ração foram fornecidas à vontade em comedouros e bebedouros automáticos. O RIFM utilizado em ambos os experimentos continha $92,66 \%$ de matéria seca, $1,32 \%$ de proteína bruta, $10,67 \%$ de fibra bruta, $6,63 \%$ de cinza, $2753 \mathrm{kcal}$ de energia digestível e $2621 \mathrm{kcal}$ de energia metabolizável $/ \mathrm{kg}$.

\section{RESULTADOS E DISCUSSÃO}

\section{Experimento 1}

Os resultados deste experimento estão na Tabela 2. A inclusão dos níveis de RIFM na dieta

TABELA 1. Composição centesimal das rações experimentais.

\begin{tabular}{|c|c|c|c|c|c|c|c|c|}
\hline \multirow[t]{3}{*}{ Ingredientes } & \multicolumn{8}{|c|}{ Níveis de inclusão do RIFM $(\%)^{1}$} \\
\hline & \multicolumn{4}{|c|}{ Experimento 1} & \multicolumn{4}{|c|}{ Experimento 2} \\
\hline & 0 & 6.67 & 13.33 & 20.00 & 0 & 10 & 20 & 30 \\
\hline Milho & 67.13 & 59.63 & 52.16 & 44.68 & 73.41 & 62.18 & 50.96 & 39.74 \\
\hline Farelo de soia & 28,09 & 29,29 & 30.48 & 31,67 & 21,80 & 23.59 & 25,38 & 27,17 \\
\hline RIFM & 0.00 & 6.67 & 13.33 & 20.00 & 0.00 & 10.00 & 20.00 & 30.00 \\
\hline $\mathrm{ODS}^{2}$ & 1.29 & 1.29 & 1.29 & 1.29 & 1.28 & 1.28 & 1.28 & 1.28 \\
\hline Fosfato bicálcico & 1,04 & 1,04 & 1,04 & 1,04 & 0,42 & 0.51 & 0.60 & 0.69 \\
\hline Calcário calcítico & 0,86 & 0,81 & 0,75 & 0,69 & 1,06 & 0,91 & 0,75 & 0.59 \\
\hline $\mathrm{PMV}^{3}$ & 0.31 & 0.31 & 0.31 & 0.31 & 0.20 & 0.20 & 0.20 & 0.20 \\
\hline Sal & 0.30 & 0.30 & 0.30 & 0.30 & 0.30 & 0.30 & 0.30 & 0.30 \\
\hline Vermífugo & 0,015 & 0,015 & 0,015 & 0,015 & 0.015 & 0.015 & 0.015 & 0,015 \\
\hline BHT & 0.01 & 0.01 & 0.01 & 0.01 & 0.01 & 0.01 & 0.01 & 0.01 \\
\hline Caulim & 0.96 & 0.64 & 0.32 & 0.00 & 1.50 & 1.00 & 0.50 & 0.00 \\
\hline Total & 100 & 100 & 100 & 100 & 100 & 100 & 100 & 100 \\
\hline \multicolumn{9}{|c|}{ Comnosicão calculada } \\
\hline Proteína bruta (\%) & 16.00 & 16.00 & 16.00 & 16.00 & 14.00 & 14.00 & 14.00 & 14.00 \\
\hline $\mathrm{ED}(\mathrm{kcal} / \mathrm{kg})$ & 3400 & 3400 & 3400 & 3400 & 3400 & 3400 & 3400 & 3400 \\
\hline Fibra bruta (\%) & 3,02 & 3,64 & 4,25 & 4,87 & 2,81 & 3,73 & 4,65 & 5.58 \\
\hline $\mathrm{Ca}(\%)$ & 0.60 & 0.60 & 0.60 & 0.60 & 0.50 & 0.50 & 0.50 & 0.50 \\
\hline $\mathrm{P}$ total $(\%)$ & 0.53 & 0.52 & 0.51 & 0.50 & 0.40 & 0.40 & 0.40 & 0.40 \\
\hline Lisina (\%) & 0.83 & 0.84 & 0.86 & 0.88 & 0.69 & 0.72 & 0.74 & 0.77 \\
\hline Custo $(\mathrm{R} \$ / \mathrm{kg})^{4}$ & 0.135 & 0.134 & 0.133 & 0.131 & 0.125 & 0.126 & 0.127 & 0.128 \\
\hline \multicolumn{9}{|c|}{ 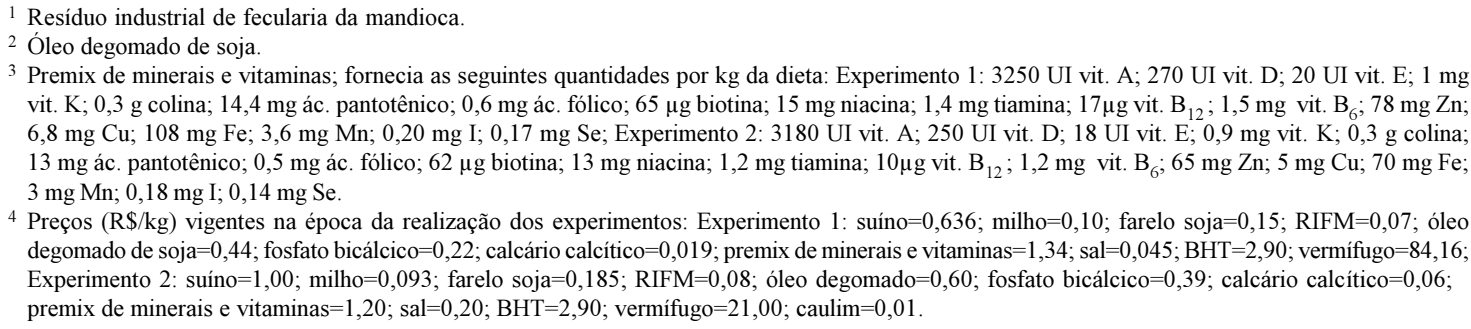 } \\
\hline
\end{tabular}


TABELA 2. Efeitos dos níveis de resíduo industrial de fecularia da mandioca (RIFM) da dieta sobre o desempenho produtivo e econômico de suínos em crescimento (experimento 1) e terminação (experimento 2 ).

\begin{tabular}{|c|c|c|c|c|}
\hline \multirow[t]{3}{*}{ Variáveis $^{1}$} & \multicolumn{4}{|c|}{ Experimento 1} \\
\hline & \multicolumn{4}{|c|}{ Níveis de inclusão do RIFM (\%) } \\
\hline & 0 & 6.67 & 13.33 & 20 \\
\hline PMI & $21.89+1.80$ & $21.54+1.16$ & $22.35+2.24$ & $21.66+1.17$ \\
\hline GPMD $^{2}$ & $804 a+73$ & $657 b+60$ & $716 b+53$ & $652 b+61$ \\
\hline $\mathrm{CRMD}^{3}$ & $2091 a+311$ & $1697 b+250$ & $1824 a b+330$ & $1708 b+162$ \\
\hline $\mathrm{CA}$ & $2,61+0,35$ & $2.58+0.31$ & $2,53+0,30$ & $2,64+0,42$ \\
\hline $\mathrm{PMFC}^{4}$ & $60.48 a+5.19$ & $53.05 b+3.61$ & $56.70 \mathrm{~b}+3.21$ & $52.96 b+2.73$ \\
\hline $\mathrm{PMA}^{5}$ & $99.46 a+2.14$ & $90.66 b+3.33$ & $91.94 b+6.10$ & $91.53 b+4.67$ \\
\hline \multirow[t]{4}{*}{$\mathrm{INBE}^{6}$} & 383 & 304 & 324 & 301 \\
\hline & \multicolumn{4}{|c|}{ Exnerimento 2} \\
\hline & \multicolumn{4}{|c|}{ Niveis de inclusão do RIFM (\%) } \\
\hline & 0 & 10 & 20 & 30 \\
\hline PMI & $54.54+2.35$ & $54.55+2.30$ & $54.18+2.68$ & $54.40+2.69$ \\
\hline GPMD & $890+59$ & $930+48$ & $919+68$ & $887+93$ \\
\hline CRMD & $2835+261$ & $2881+81$ & $2923+319$ & $2822+453$ \\
\hline $\mathrm{CA}$ & $3,18+0,20$ & $3,10+0,14$ & $3,19+0,42$ & $3,18+0,39$ \\
\hline PMF & $91.96+3.44$ & $93.69+3.50$ & $92.88+4.62$ & $91.67+4.95$ \\
\hline INBE & 506 & 538 & 519 & 500 \\
\hline
\end{tabular}

1 PMI: peso médio inicial (kg); GPMD: ganho de peso médio diário (g); CRMD: consumo de ração médio diário (g); CA: conversão alimentar; PMFC: peso médio ao final do crescimento $(\mathrm{kg})$; PMA: peso médio ao abate $(\mathrm{kg})$; INBE: índice nutricional bioeconômico $(\mathrm{g})$; PMF: peso médio final $(\mathrm{kg})$.

2 Efeito cúbico $(\mathrm{P}<0,01)\left(\mathrm{R}^{2}=0,80, \mathrm{y}=804-54,06 \mathrm{x}+6,03 \mathrm{x}^{2}-0,19 \mathrm{x}^{3}\right)$.

${ }^{3}$ Efeito linear $(\mathrm{P}<0,04)\left(\mathrm{R}^{2}=0,74, \mathrm{y}=2090-136 \mathrm{x}\right)$.

${ }^{4}$ Efeito cúbico $(\mathrm{P}<0,01)\left(\mathrm{R}^{2}=0,79, \mathrm{y}=60,47-2,87 \mathrm{x}+0,33 \mathrm{x}^{2}-0,01 \mathrm{x}^{3}\right)$.

${ }^{5}$ Efeito quadrático $(\mathrm{P}<0,05)\left(\mathrm{R}^{2}=0,69, \mathrm{y}=98,88-1,28 \mathrm{x}+0,05 \mathrm{x}^{2}\right)$.

${ }^{6}$ Efeito linear $(\mathrm{P}<0,03)\left(\mathrm{R}^{2}=0,72, \mathrm{y}=362-3,40 \mathrm{x}\right)$.

dos suínos em crescimento provocou efeito cúbico sobre o PMFC $\left(\mathrm{P}<0,01, \mathrm{R}^{2}=0,79\right)$ e o GPMD $(\mathrm{P}<0,01$, $\left.\mathrm{R}^{2}=0,80\right)$, e efeito linear $\left(\mathrm{P}<0,04, \mathrm{R}^{2}=0,74\right)$ sobre o CRD. OPMA foi afetado de forma quadrática $(\mathrm{P}<0,05$, $\left.\mathrm{R}^{2}=0,69\right)$ pelos níveis de inclusão do RIFM na dieta. A CA não foi afetada $(\mathrm{P}>0,10)$ pelos tratamentos. Através do INBE, verificou-se uma redução linear $\left(\mathrm{P}<0,03, \mathrm{R}^{2}=0,72\right)$ do ganho de peso líquido com o aumento dos níveis de RIFM na dieta.

A redução no ganho de peso foi, em média, de $15,9 \%$ nos tratamentos com RIFM, e foi causada por redução do consumo de alimento (em média $16,8 \%$ ); não houve efeito sobre a conversão alimentar. A palatabilidade do RIFM pode ter sido uma das causas da redução do consumo das rações que continham este ingrediente. Creswell (1978), em sua revisão bibliográfica, enfatizou que a natureza pulverulenta da mandioca tem sido mencionada por vários autores como causadora de baixa palatabilidade.

Outra causa da redução do consumo pode ter sido o pior balanceamento dos aminoácidos nas dietas que continham raspa de mandioca, já que o nível de lisina da dieta aumentou de 0,83 para $0,88 \%$ e o nível de metionina + cistina diminuiu de 0,49 para $0,46 \%$, à medida que se aumentou o nível de RIFM na dieta. Segundo o National Research Council (1988), a redução do consumo de alimento é um sinal primário de deficiência de aminoácidos. Em um trabalho desenvolvido por Zoby et al. (1971), a substituição total do milho por RIFM provocou redução de $17,9 \%$ no ganho de peso e piorou em $26,6 \%$ a conversão alimentar, mas não afetou o consumo de alimento, em dietas com o mesmo nível de extrato etéreo, até os $50 \mathrm{~kg}$ de peso vivo. Pela suplementação com metionina foi melhorado o ganho de peso, 
tornando-o semelhante ao tratamento testemunha, e a conversão alimentar foi melhorada em $7,1 \%$, além de aumentar em $15 \%$ o consumo de alimento.

Embora o nível mais baixo de metionina + cistina (no maior nível de inclusão do RIFM) ainda esteja acima do recomendado pelo National Research Council (1988), para a fase de crescimento $(0,41 \%)$ provavelmente as exigências desse aminoácido para os animais utilizados neste experimento são superiores a este valor. Além disso, deve-se considerar que ao incluir o RIFM nas dietas, o farelo de soja passou a suprir uma maior proporção da proteína da dieta, reduzindo a participação da proteína do milho. Com isso, substituiu-se uma proteína cuja digestibilidade ileal da lisina é de $68 \%$ e a da metionina é $85 \%$, por outra em que a digestibilidade ideal da lisina é de $83 \%$ e a da metionina é de $83 \%$. Dessa forma, podese observar que o desequilíbrio entre os aminoácidos é ainda maior quando se consideram os valores de aminoácidos digestíveis, e que a suplementação com metionina ou aumento do nível de proteína da dieta poderão ser necessários para garantir os níveis mínimos requeridos de metionina + cistina, quando se utilizar RIFM na dieta de suínos em crescimento. Assim, é provável que não haja efeito negativo sobre o desempenho.

A redução do ganho de peso verificada neste experimento está de acordo com a encontrada em um experimento com suínos em crescimento-terminação (Nicolaiewsky et al., 1986a), em que também foram fornecidas dietas com baixos níveis de inclusão do RIFM (a partir de 7,5\%). Em outro experimento, em que foram fornecidos níveis de raspa residual de mandioca de 0,30 e $45 \%$ da dieta para suínos em crescimento e 0 e $45 \%$ para os mesmos suínos em terminação (Nicolaiewsky et al., 1986b), os autores observaram redução de ganho de peso na fase de crescimento a partir do nível $30 \%$, embora a diferença somente tenha sido significativa no nível de $45 \%$.

O menor ganho de peso dos suínos que receberam as dietas contendo qualquer um dos níveis de inclusão do RIFM no crescimento resultou em menor peso ao abate, o que indica que, mesmo após receber uma dieta baseada em milho e farelo de soja no período de terminação, esses animais não conseguiram compensar o baixo ganho de peso anterior, e ainda tiveram seu desempenho prejudicado, pois as diferenças entre os tratamentos aumentaram nesse período (Tabela 2). Os valores mínimos de PMA estimados através da equação quadrática foram obtidos no nível de $12,8 \%$ de inclusão do RIFM na dieta.

A redução do INBE com a inclusão do RIFM na dieta deve-se ao baixo ganho de peso nestes tratamentos, já que o custo destas dietas foi reduzido com o aumento do nível do RIFM, levando-se em conta os preços dos ingredientes na época da realização deste trabalho (Tabela 1). Em média, o INBE diminuiu em 19\% com a inclusão dos diferentes níveis de RIFM, ou 3,4 g para cada unidade percentual de inclusão do RIFM na dieta, de acordo com a equação: $y=362-3,40 x$. No entanto, estes resultados são válidos somente para os preços citados na Tabela 1.

\section{Experimento 2}

Os diferentes níveis de inclusão do RIFM na dieta não afetaram significativamente $(\mathrm{P}>0,10)$ o desempenho, de acordo com o observado no GPMD, PMF, CRMD e CA (Tabela 2). Houve um aumento apenas numérico no GPMD nos níveis de 10 e 20\% de inclusão do RIFM na dieta, causado por um aumento equivalente no CRMD.

Em um experimento com suínos em terminação, onde foram testados níveis de $0,15,30,45$ e $60 \%$ de raspa residual de mandioca na dieta, Nicolaiewsky et al. (1986b) também não verificaram diferença significativa no desempenho até o nível de $45 \%$, embora tenha havido redução de $6,4 \%$ no ganho de peso, a partir do nível $30 \%$. O consumo de alimento não foi afetado, e a conversão alimentar sofreu efeito negativo significativo somente no nível $60 \%$.

Os níveis de metionina + cistina das dietas nessa fase foram reduzidos de 0,45 a $0,40 \%$ e os níveis de lisina aumentaram de 0,69 a $0,77 \%$ com o aumento dos níveis de RIFM. Apesar disso, acredita-se que os níveis de metionina tenham sido suficientes para cobrir as necessidades dos suínos, pelo fato de não ter ocorrido resposta negativa sobre o desempenho. Com a substituição total do milho por raspa residual de mandioca, no trabalho desenvolvido por Zoby et al. (1971), dos 50 aos $95 \mathrm{~kg}$ de peso vivo não houve efeito sobre o ganho de peso, mas a conversão 
alimentar piorou em 8,3\%. A suplementação com metionina nessa fase não melhorou nenhuma das variáveis de desempenho. De acordo com estes autores, os níveis de metionina de todas as dietas foram suficientes para atender os requerimentos daqueles animais na fase de terminação, ao contrário do que ocorreu na fase de crescimento.

Através do INBE, verificou-se que não houve redução significativa no custo da alimentação com a utilização do RIFM (Tabela 2), considerando-se os preços vigentes na época da realização do trabalho (Tabela 1). Como o desempenho não foi afetado pela inclusão do RIFM na dieta, a redução do custo da alimentação dependeria somente da redução do custo da dieta. Uma redução no custo das dietas com a utilização do RIFM dependeria principalmente de reduções no preço do farelo de soja, cuja participação na dieta aumenta com a inclusão do RIFM, ou de aumentos no preço do milho.

Coursey \& Halliday (1974) observaram que, em função do aumento na necessidade de suplementação com ingredientes protéicos nas dietas contendo mandioca, em geral é mais atrativo economicamente incluir a mandioca nas dietas para as fases de terminação e reprodução, as quais não necessitam de um conteúdo de proteína muito alto.

\section{CONCLUSÕES}

1. A inclusão de resíduo industrial de fecularia da mandioca na dieta de suínos em crescimento a partir do nível de $6,67 \%$ provoca redução do desempenho.

2. A inclusão de até $30 \%$ de resíduo industrial de fecularia da mandioca na dieta de suínos em terminação não afeta o desempenho desses animais.

\section{REFERÊNCIAS}

ANUÁRIO ESTATÍSTICO DO BRASIL. Rio de Janeiro: IBGE, 1995. 864p.

CENTRO INTERNACIONAL DE AGRICULTURA TROPICAL. (Cali, Colombia). Sistemas de producción de ganado porcino. Informe anual. CIAT, Cali, 1970. p.25-34.
CENTRO INTERNACIONAL DE AGRICULTURA TROPICAL. (Cali, Colombia). Unidad de porcinos. Informe anual. CIAT, Cali, 1978. p.E1-E20.

COURSEY, D.G.; HALLIDAY, D. Cassava as animal feed. Outlook on Agriculture, London, v.8, n.1, p.1014, 1974.

CRESWELL, D.C. Cassava (Manihot esculenta Crantz) as a feed for pigs and poultry - A review. Tropical Agriculturist, Trinidad, v.55, n.3, p.273-282, 1978.

EMBRAPA. Centro Nacional de Pesquisa de Suínos e Aves (Concórdia, SC). Tabela de composição química e valores energéticos de alimentos para suínos e aves. 3.ed. Concórdia, 1991. 97p. (Embrapa-CNPSA. Documentos, 19).

GOMEZ, G. Cassava as a swine feed. World Animal Review, Roma, v.29, p.13-20, 1979.

GUIDONI, A.L.; GODOI, C.R.M.; BELLAVER, C. Uso do índice nutricional bio-econômico como medida do desempenho nutricional animal. In: REUNIÃO ANUAL DA SOCIEDADE BRASILEIRA DE ZOOTECNIA, 31., 1994, Maringá. Anais... Maringá: SBZ, 1994. p.32.

MULLER, Z.; CHOU, K.C.; NAH, K.C. La yuca como sustituto total de los cereales en las raciones del ganado y de las aves de corral. Revista Mundial de Zootecnia, Roma, v.12, p.19-24, 1974.

NATIONAL RESEARCH COUNCIL. (Washington, DC) Nutrient requirements of swine. Washington, DC: National Academy of Science, 1988. 64 p. (Nutrient Requirements of Domestic Animals, 2).

NICOLAIEWSKY, S.; D'AGOSTIN, J.; CAETANO, L.A.P. Níveis crescentes de resíduo de mandioca (raspa) na alimentação de suínos em crescimento e terminação. In: REUNIÃO ANUAL DA SOCIEDADE BRASILEIRA DE ZOOTECNIA, 23., 1986, Campo Grande. Anais... Campo Grande: SBZ, 1986a. p.49.

NICOLAIEWSKY, S.; D'AGOSTIN, J.; CAETANO, L.A.P. Resíduo de mandioca (raspa) na alimentação de suínos em crescimento e terminação. In: REUNIÃO ANUAL DA SOCIEDADE BRASILEIRA DE ZOOTECNIA, 23., 1986, Campo Grande. Anais... Campo Grande: SBZ, 1986b. p.48.

SAS Institute. (Cary, NC). Guide for personal computers: version 6. Cary, NC, 1990. 1028p.

ZOBY, J.L.F.; CAMPOS, J.; MAYROSE, V.; COSTA, P.M.A. Raspa de mandioca com suplementação de gordura e metionina, na alimentação de suínos. Revista Ceres, Viçosa, v.18, n.97, p.195-209, 1971. 